\title{
Ventilator-Associated Pneumonia is Linked to a Worse Prognosis Than Community-Acquired Pneumonia in Children
}

\section{Maria Hernandez}

Hospital Sant Joan de Déu Barcelona

Mònica Girona-Alarcon

Hospital Sant Joan de Déu Barcelona

Sara Bobillo-Perez ( $\nabla$ sbobillo@sjdhospitalbarcelona.org)

Hospital Sant Joan de Déu Barcelona

\section{Mireia Urrea}

Hospital Sant Joan de Déu Barcelona

\section{Anna Sole-Ribalta}

Hospital Sant Joan de Déu Barcelona

\section{Mònica Balaguer}

Hospital Sant Joan de Déu Barcelona

Francisco-José Cambra

Hospital Sant Joan de Déu Barcelona

lolanda Jordan

Hospital Sant Joan de Déu Barcelona

\section{Research Article}

Keywords: Ventilator-associated pneumonia (VAP), Paediatric Intensive Care Units (PICU), communityacquired pneumonia (CAP)

Posted Date: January 23rd, 2021

DOl: https://doi.org/10.21203/rs.3.rs-149977/v1

License: (c) (i) This work is licensed under a Creative Commons Attribution 4.0 International License.

Read Full License 


\section{Abstract}

\section{Background}

Around $12-20 \%$ of patients with community-acquired pneumonia (CAP) require critical care. Ventilatorassociated pneumonia (VAP) is the second cause of nosocomial infection in Paediatric Intensive Care Units (PICU). As far as we know, there are no studies comparing both types of pneumonia in children, thus it remains unclear if there are differences between them in terms of severity and outcomes.

\section{Objective}

The aim was to compare clinical and microbiological characteristics and outcomes of patients with severe CAP and VAP.

\section{Methods}

A retrospective descriptive study, including patients diagnosed of VAP and CAP, with a positive respiratory culture and under mechanical ventilation, admitted to the PICU from 2015 to 2019.

\section{Results}

238 patients were included; 163 (68.4\%) with CAP, and 75 (31.5\%) with VAP. Patients with VAP needed longer mechanical ventilation ( 14 vs. 7 days, $p<0.001)$ and more inotropic support ( 49.3 vs. $30.7 \%$, $\mathrm{p}=0.006)$. Patients with VAP had higher mortality ( 12 vs. $2.5 \%, p=0.005)$.

Enterobacteriaceae were more involved with VAP than with CAP (48 vs. $9 \%, \mathrm{p}<0.001$ ). Taking into account only de non-drug sensitive microorganisms, patients with VAP tended to have more multidrug-resistant bacteria (30 vs. $10.8 \%, p=0.141$ ) than patients with CAP.

\section{Conclusion}

Patients with VAP had worse prognosis than patients with CAP, needing longer mechanical ventilation, more inotropic support and had higher mortality. Patients with VAP were mainly infected by Enterobacteriaceae and had more multidrug resistant microorganisms than patients with CAP.

\section{Introduction}

Pneumonia is one of the most common causes of infection requiring hospitalisation in children and it is the most frequent reason for antibiotic use in paediatric hospitals ${ }^{1,2}$. Pneumonia in children can be classified as either community-acquired pneumonia (CAP) or ventilator-associated pneumonia (VAP). Optimising the selection of appropriate antimicrobial therapies can be difficult for paediatric pneumonia because of the ill-defined diagnostic criteria and the difficulty in differentiating viral and bacterial aetiology ${ }^{3}$. 
CAP is currently one of the primary causes of mortality in children worldwide, especially in those under 5 years old ${ }^{4,5}$. Although mortality is lower in developed countries, CAP is still associated with substantial morbidity and remains the most common indication for paediatric hospitalisation outside the newborn period in the United States ${ }^{1}$.

Moreover, about $12-20 \%$ of paediatric CAP cases require critical care ${ }^{1,3,6}$, primarily due to the development of respiratory failure requiring assisted ventilation and pneumonia complicated by septicaemia ${ }^{4}$. Risk factors that contribute to developing severe CAP are the presence of underlying comorbidities, including prematurity, low birth weight, bronchopulmonary dysplasia, asthma or mucusclearance disorders (such as cystic fibrosis), congenital heart disease, immunodeficiency, and severe cerebral palsy. Another risk factor is a relevant medical history of severe pneumonia (inpatient stay requiring oxygen, complications such as a lung abscess, effusion, empyema, or recurrent pneumonia) ${ }^{4,7}$.

Among the different hospital-acquired infections in children, VAP is the most common after blood stream infections. In paediatric intensive care units (PICUs), patients on mechanical ventilation (MV) run the risk of developing VAP, which is estimated to occur in around $10-20 \%$ of ventilated patients. As is the case with CAP, VAP involves high morbidity and mortality; it can prolong the length of respiratory support and hospitalisation, increase the mortality rate, and directly affect patient outcomes in PICUs ${ }^{3,8,9}$. Age less than 1 year, altered immune status, unplanned emergency intubations and reintubation, acute respiratory distress syndrome, continuous enteral feeding and use of discontinuous sedation have been associated with an increased risk of developing VAP ${ }^{8-10}$

Severe CAP requiring admission to the PICU and VAP differ not only from an aetiological and pathophysiological point of view, but also as regards their microbiological findings. Despite the fact that the most common microorganisms related to paediatric pneumonia are viruses, mainly in children under 5 years old, identifying the most common bacterial pathogens is important to aid in decisions related to empirical antibiotics ${ }^{11}$.

It is known that the bacterial pathogens most frequently related to CAP are gram positive bacteria and non-Enterobacteriaceae gram negative bacteria, especially S. pneumoniae, S. aureus, $H$. influenzae and $M$. catarrhalis $^{12,13}$. By contrast, the most frequent bacterial pathogens isolated in VAP are Enterobacteriaceae and other gram negative bacteria ${ }^{9}$. Foglia et at. described that early-onset VAP is usually caused by $M$. catarrhalis, $H$. influenzae or $S$. pneumoniae, but late-onset VAP is often caused by gram negative species or $S$. aureus, including methicillin-resistant $S$. aureus (MRSA) and other resistant bacteria ${ }^{14}$. Regarding the susceptibility pattern, it has been reported that multidrug and extensively drugresistant microorganisms are more common among patients with VAP ${ }^{15}$. Due to this, dealing with VAP in any intensive care unit is challenging. It is important to identify the burden of VAP in any setting, so that prevention strategies can be implemented and strengthened.

As far as we know, there are no published references in paediatrics comparing VAP and CAP in terms of the risk factors, the microorganisms related to them, and the outcomes. Our hypothesis was that CAP and 
VAP in critically ill children have the same severity. Therefore, we decided to analyse the differences between them in a paediatric intensive care unit (PICU). The main objective of the study was to describe the clinical and microbiological characteristics of severe CAP and VAP. The secondary objective was to compare the different outcomes depending on the type of pneumonia.

\section{Material And Methods}

This was a single-centre retrospective and observational study performed in the PICU of Hospital Sant Joan de Déu in Barcelona, which is a tertiary referral paediatric hospital with 326 beds and approximately 1,200 PICU admissions per year.

Patients under 18 years old admitted to the PICU from January 2015 to December 2019 who required MV with a confirmed diagnosis of VAP or CAP were included. Patients without a positive respiratory culture or those with less than $10^{5}$ colonies $/ \mathrm{mL}$ were excluded in order to include neither colonisations nor contaminations.

Patients were classified depending on their diagnosis, according to following definitions included in the CDC criteria:

CAP: According to the recent criteria published by Shah et al. ${ }^{16}$, this is the presence of signs and symptoms of pneumonia in a previously healthy child caused by an infection that has been acquired outside of the hospital. Signs and symptoms of pneumonia include clinical data (fever $\geq 38^{\circ} \mathrm{C}$, tachypnoea, nasal flaring, grunting, retractions, hypoxia), chest X-ray opacities, and increased levels of acute phase reactants (CRP $\geq 70 \mathrm{mg} / \mathrm{dL}$ and/or PCT $\geq 1 \mathrm{ng} / \mathrm{mL}$ ) ${ }^{17-19}$. Patients needed to fulfill all 3 diagnostic criteria. A microbial diagnosis required a positive respiratory culture and/or a positive blood culture (excluding microorganisms found on the skin) and/or a positive PCR for pneumoniae or $S$. aureus.

VAP: Pneumonia where the patient is on mechanical ventilation for at least 2 calendar days on the date of diagnosis, with the day they were placed on the ventilator being day 1 AND the ventilator being in place on the date of diagnosis or the day before. Diagnosing VAP requires clinical data (worsening respiratory assessment), radiological findings (consolidations on X-ray or worsening thereof), and a positive culture (tracheal aspirate, $\geq 10^{5} \mathrm{CFU} / \mathrm{mL}$ ) ${ }^{11,20,21}$.

The study was carried out in accordance with the Spanish regulations. The study was approved by the Sant Joan de Déu Hospital Clinical Research Ethics Committee (PIC-180-19). Informed consent was not required due to the retrospective and non-interventional design of the study, according to the Ethics Committee. The study was carried out in compliance with the Declaration of Helsinki.

\section{Variables}

The following demographic data were collected from the electronic medical records: age, gender, comorbidities, Paediatric Risk of Mortality Score (PRISM III) ${ }^{22}$ at admission, and reason for admission 
(respiratory, cardiovascular, neurologic, haemato-oncological, surgical, sepsis). Risk factors for VAP were considered as the days in the hospital until intubation, days in the hospital until infection, and days on MV until infection, considering infection when the previously described criteria to define VAP were met. The antibiotic therapy used was recorded (including duration and the need to switch medications). The microbiological data collected included the microorganisms isolated in the blood culture and respiratory cultures and their antibiotic susceptibility (defined according to European Centre for Disease Prevention and Control criteria) ${ }^{20}$. As for the latter measure, bacteria were classified as either drug-susceptible (DS) or non-drug-susceptible (non-DS). The non-DS group included drug resistant (DR, if non-susceptible to at least 1 agent in less than 3 categories) and multidrug-resistant (MDR, if non-susceptible to at least 1 agent in 3 or more categories) ${ }^{23}$. Specific resistance phenotypes, such as extended-spectrum betalactamase (ESBL) and MRSA were also recorded. Outcomes were considered as the support required during the PICU admission, including the duration of the respiratory support (days on MV or non-invasive mechanical ventilation), the inotropic support in the PICU (requirement, duration, and the maximum vasoactive-inotropic score, VIS) ${ }^{24}$, the need for extracorporeal support, the length of stay in the PICU and in the hospital, and the mortality during the their stay in the PICU. The analytical biomarkers recorded were $\mathrm{C}$-reactive protein and procalcitonin, analysing the highest value of each one.

\section{Statistical Analysis}

The statistical analysis was performed using IBM SPSS 25.0 Statistics ${ }^{\circledR}$. Categorical variables were indicated as frequency $(n)$ and percentage $(\%)$, whereas continuous variables were summarised as median and interquartile range (IQR) because they were not normally distributed. The comparison of categorical variables was performed using the $\chi 2$-test or Fisher's exact test. Continuous variables were compared with the Mann-Whitney U test. Probability values of less than 0.05 were considered statistically significant.

\section{Results}

\section{Clinical characteristics}

In total, 238 patients were included; 125 (52.5\%) were males and the median age was 6.3 months (IQR 1.6-43.5). A total of 100 patients (42\%) had some comorbidity. Among all the patients, $163(68.4 \%)$ were diagnosed with CAP and 75 (31.5\%) with VAP. The main demographical data are detailed in Table 1.

Comparing patients with CAP vs. those with VAP, no differences were found as regards gender or age. Patients with VAP had a higher percentage of associated comorbidity ( $64 \% \mathrm{vs.} 31.9 \%, p<0.001$ ), and they were also more likely to have tracheostomies ( $8 \%$ vs. $2 \%, p=0.013$ ). Admission due to respiratory problems was more frequent in patients with CAP than in patients with VAP $(70.6 \%$ vs. $46.7 \%, p=0.001)$. Patients who developed VAP were admitted due to cardiovascular reasons more frequently than patients with CAP $(20 \%$ vs. $4.9 \%, p<0.001)$. 
While the diagnosis of CAP was made at day 1 (IQR 0-3), VAP was diagnosed at day 11 (IQR 7-14), $p=0.001$. This difference was also significant when considering the days elapsed from the endotracheal intubation to the infection ( 1 vs. 8 days, $p=0.001$ ).

No differences were found in C-reactive protein values (103 vs. $86.6 \mathrm{mg} / \mathrm{L}, p=0.243$ ) nor in procalcitonin levels (1.7 vs. $2.2 \mathrm{ng} / \mathrm{mL}, p=0.191$ ). Patients with VAP required longer antibiotic treatment (10 vs. 7 days, $p<0.001)$ and more antibiotic switching (63.5\% vs. $45.7 \%, p=0.011)$.

Patients with VAP had a higher proportion of sepsis than patients with CAP ( $24 \%$ vs. $14.7 \%$ ), even though the difference was not statistically significant $(p=0.081)$.

As regards the microbiological data, Enterobacteriaceae were the microorganisms most frequently associated with VAP $(n=36,48 \%)$, yielding a higher percentage than those patients with CAP $(n=5.5,9 \%$, $p<0.001)$. The main microorganisms isolated in CAP were gram negative bacteria $(n=94,57.7 \%)$, followed by gram positive bacteria $(\mathrm{n}=60,36.8 \%)$, both in higher proportions than in $\operatorname{VAP}(p=0.05$ and $p<0.001$, respectively). Looking at the specific microorganisms, CAP was more frequently related to Haemophilus spp. $(p<0.001), S$. pneumoniae $(p<0.001), S$. aureus $(p=0.021)$, and $M$. catarrhalis $(p=0.010)$ than VAP. VAP was more likely to be associated with $P$. aeruginosa $(p<0.001)$, Klebsiella spp. $(p<0.001)$, Enterobacter spp. $(p<0.001)$, E. coli $(p=0.030)$, S. maltophilia $(p=0.003)$, and Serratia $\operatorname{spp}(p=0.009)$ than CAP.

No differences were found in the percentage of DS microorganisms between groups $(p=0.505)$. Taking into account only the non-DS microorganisms, patients with CAP tended to have more DR bacteria ( $86.5 \%$ vs. $65 \%, p=0.058)$ while patients with VAP tended to have more MDR (30\% vs. $10.8 \%, p=0.141)$ and ESBL ( $30 \%$ vs. $8.1 \%, p=0.054)$ ones, even though the differences were not statistically significant. The main microbiological data are detailed in Table 2.

\section{Outcomes}

Patients with VAP required prolonged respiratory support: they needed more days of MV (14 vs. 7 days on $\mathrm{MV}, p<0.001$ ), and non-invasive ventilation (3 vs. 2 days, $p<0.001$ ).

Patients with VAP needed inotropic support more often than patients with CAP ( $49.3 \%$ vs. $30.7 \%$, $p=0.006$ ) and for a longer period ( 8 vs. 3 days, $p<0.001$ ). They tended to require higher levels of inotropic treatment when comparing VIS score ( 15 vs. $10, p=0.073$ ), even though the difference was not statistically significant. Table 3 summarizes the main outcomes for the two groups.

Patients with VAP had a longer stay in the PICU (24 vs. 12 days, $p<0.001)$ and in the hospital ( 35.5 vs. 19 days, $p<0.001)$ than patients with CAP. They had a more frequent need for extracorporeal support $(10.7 \%$ vs. $1.8 \%, p=0.005)$ and had higher mortality rate $(12 \%$ vs. $2.5 \%, p=0.005)$.

\section{Discussion}


To the best of our knowledge, there is no data available comparing the risk factors, outcomes, and microbiological characteristics of severe CAP and VAP in the paediatric population. In this study, we have analysed data from the last five years on children admitted to the PICU with pneumonia, both CAP and VAP. It has revealed some data that is of interest to clinicians, since these are both associated with a high mortality rate, especially VAP, in which mortality can reach up to $20 \% 25$.

The main objective of the present study was to analyse the differences between CAP and VAP in children. According to these results, there are important differences between both diseases, since patients with VAP had worse prognosis than patients with CAP, needing longer mechanical ventilation, more inotropic support and had higher mortality. Moreover, patients with VAP were mainly infected by Enterobacteriaceae and had more multidrug resistant microorganisms.

Patients that developed VAP were more prone to having comorbidities than patients with CAP. This is consistent with other studies in adults, in which underlying diseases and comorbidities have been described as risk factors for developing VAP ${ }^{25}$. In addition, patients that developed VAP were more likely to have a tracheostomy than those with CAP. Since tracheostomised children are at an increased risk of developing pneumonia, it is important to implement preventative care bundle measures in these vulnerable patients when they're admitted to the PICU ${ }^{26}$.

Moreover, patients with VAP were admitted due to a cardiovascular disease in the $20 \%$ of the cases, which was higher than in patients with CAP. The vulnerability of patients with cardiovascular diseases has been reported in other studies ${ }^{27}$, as they are more susceptible to developing VAP and, additionally, nosocomial infections in these patients are an important cause of morbidity and mortality. In some studies performed on adults, they even propose decontamination or pre-emptive antibiotic therapy in order to prevent the development of VAP ${ }^{28,29}$.

While patients with CAP were diagnosed with infection at admission, patients that ended up with VAP received this diagnosis later (after 11 days). This fact highlights that even patients that do not develop pneumonia initially are at a high risk of developing it if they are on MV. For this reason, strategies to prevent VAP such as the elevation of the head of the bed, regular oral care, maintaining ventilator circuits, the use of cuffed endotracheal tubes, and minimising the duration of MV are highly recommended ${ }^{30}$.

Patients with VAP had higher inotropic requirements, longer MV, a longer length of stay, and required more extracorporeal support than patients with CAP. Furthermore, the mortality was higher in patients with VAP than in patients with CAP. Kollef et al. described in a recent multicentre study that patients with VAP seem to have worse outcomes than patients with CAP, in terms of mortality rate and length of stay ${ }^{31}$. Therefore, even if both types of patients are in a critical care unit and on MV, we should be especially concerned about the ones with VAP, since they are more likely to require more support and have a poorer outcome. 
As for the microbiological data, remarkable differences were found between the two groups. The bacteria most frequently involved with CAP were gram negative species, especially Haemophilus spp, followed by gram positive species such as $S$. pneumoniae and $S$. aureus. In children, CAP is usually caused by a virus, followed by $S$. pneumoniae and others like Haemophilus spp and $S$. aureus ${ }^{32}$. In recent years, the development of vaccines against $S$. pneumoniae (the 13-valent pneumococcal conjugate vaccine) and Haemophilus influenzae type $B$ has helped to decrease the incidence of CAP related to these microbes, especially severe cases of CAP ${ }^{21}$. Furthermore, after the implementation of these vaccines, other changes in the epidemiology have been revealed: non-vaccine serotypes have been isolated in very few cases but the other hand, an increasing prevalence of CAP with viral involvement has been described 33,34. Despite this, the cases of CAP related with S. pneumoniae and Haemophilus spp are still relevant, mainly due to persistent inequities in access to healthcare, especially in low and middle-income countries 35 .

In contrast, VAP was caused mainly by Enterobacteriaceae. In adults, it has been widely described how Enterobacteriaceae are involved in a high percentage of VAP cases ${ }^{36}$. In our study, the specific microorganisms most frequently involved with VAP were Pseudomonas aeruginosa, followed by Enterobacteriaceae. As previously reported, MV duration and length of PICU stay were significantly longer in the group with VAP. Chomton et al. found that the median MV duration at VAP diagnosis was longer for VAP due to nosocomial microorganisms such as $P$. aeruginosa or $E$. coli when compared with VAP due to community-acquired bacteria such as $H$. influenzae and $S$. pneumoniae ${ }^{10}$. This fact was also explained by Kollef et al.; oropharyngeal and tracheal colonisation with Pseudomonas and enteric gram-negative bacilli increases in step with length of hospital stay and severity of illness ${ }^{37}$.

One of the major concerns worldwide nowadays is the increasing prevalence of multidrug-resistant microorganisms and the lack of new antimicrobial agents for use in paediatric pneumonia ${ }^{3}$. Patients with VAP required more days of antibiotic treatment than patients with CAP. Moreover, they were more likely to need their antibiotics to be switched. This is probably related to the differences in the type of microorganism the pneumonia involves. While CAP is usually caused by drug-sensitive bacteria and empirical treatment is normally sufficient, in VAP, as reported, there are more MDR and ESBL bacteria, and patients therefore more commonly need broad-spectrum antibiotic treatment. This observation is consistent with the results of other studies, in which ESBL has been related with VAP ${ }^{38}$. A significant morbidity and mortality associated with inadequate or delayed antibiotic treatment is reported in adults, so in children it can be assumed that adequate antibiotic use is also a key prognostic factor ${ }^{10}$.

We acknowledge several limitations in this study. Patients admitted to the Neonatal ICU ( $<1$ month) were not included (because the neonatal and paediatric ICUs are two separate units with a high inflow of patients), and therefore we do not have results for the neonatal population. In addition, it is a singlecentre study, so the results might be difficult to extrapolate to other populations. However, for a paediatric study, it has quite a large number of patients, so the results may be useful to other PICUs. 
Despite these limitations, to our knowledge this is the first study comparing the risk factors and outcomes of severe CAP and VAP in children. Therefore, we believe that it provides valuable information on the paediatric population.

To sum up, children that develop VAP seem to be more vulnerable than those with CAP, because they had a higher proportion of comorbidities and they had an increased prevalence of cardiovascular diseases. Moreover, patients with VAP required more inotropic support, longer MV, and had longer hospitalisation times than patients with CAP, and they ended up having a higher mortality than patients with CAP. In light of this, strategies to prevent nosocomial infections should be carefully executed in order to avoid VAP, since it worsens patients' prognosis. Finally, considering that VAP is usually related to Enterobacteriaceae, the correct antibiotic treatment should be implemented as soon as signs of infection appear so as to improve the outcome of the patient.

\section{Declarations}

\section{Author contributions}

Drs MH, MGA, and SBP had primary responsiblity for collecting data, and preliminary data analysis.

Drs. MGA and SBP had the responsability of writing the manuscrit and reviewing statistical analysis.

Drs. MU, ASR and MB also contributed to the writing of the manuscript

Drs. FJC and IJ supervised the design and execution of the study, performed the final data analyses and contributed to the writing of the manuscript.

\section{Competing interests}

The authors declare no competing interests

\section{References}

1. Jain, S. et al. Community-acquired pneumonia requiring hospitalization among U.S. children. N. Engl. J. Med. 372, 835-845 (2015).

2. Gerber, J. S. et al. Identifying Targets for Antimicrobial Stewardship in Children's Hospitals. Infect. Control Hosp. Epidemiol. 34, 1252-1258 (2013).

3. Dassner, A. M., Nicolau, D. P. \& Girotto, J. E. Management of Pneumonia in the Pediatric Critical Care Unit: An Area for Antimicrobial Stewardship. Curr. Pediatr. Rev. 13, 49-66 (2016).

4. Haq, I. J., Battersby, A. C., Eastham, K. \& McKean, M. Community acquired pneumonia in children. BMJ 356, 1-10 (2017).

5. Rudan, I. et al. Epidemiology and etiology of childhood pneumonia in 2010: Estimates of incidence, severe morbidity, mortality, underlying risk factors and causative pathogens for 192 countries. J. 
Glob. Health 3, (2013).

6. Myers AL, Hall M, Williams DJ, Auger K, Tieder JS, Statile A, Jerardi K, McClain L, S. S. Prevalence of Bacteremia in Hospitalized Pediatric Patients With Community-acquired Pneumonia. Pediatr Infect Dis J. 32(7), 736-740 (2013).

7. Shan, W. et al. Risk Factors for Severe Community-aquired Pneumonia among Children Hospitalized with CAP Younger Than 5 Years of Age. Pediatr. Infect. Dis. J. 38, 224-229 (2019).

8. Amanati, A. et al. Incidence of Ventilator-Associated Pneumonia in Critically III Children Undergoing Mechanical Ventilation in Pediatric Intensive Care Unit. Children 4, 56 (2017).

9. Hamid, M. H., Malik, M. A., Masood, J., Zia, A. \& Ahmad, T. M. Ventilator-associated pneumonia in children. J. Coll. Physicians Surg. Pak. 22, 155-8 (2012).

10. Chomton, M. et al. Ventilator-associated pneumonia and events in pediatric intensive care: A single center study. Pediatr. Crit. Care Med. 19, 1106-1113 (2018).

11. Harris, M. et al. British Thoracic Society guidelines for the management of community acquired pneumonia in children: Update 2011. Thorax 66, (2011).

12. Jiang, W. et al. Etiologic spectrum and occurrence of coinfections in children hospitalized with community-acquired pneumonia. BMC Infect. Dis. 17, 1-8 (2017).

13. Zhang, Q., Guo, Z., Bai, Z. \& MacDonald, N. E. A 4 year prospective study to determine risk factors for severe community acquired pneumonia in children in southern China. Pediatr. Pulmonol. 48, 390397 (2013).

14. Foglia, E., Meier, M. D. \& Elward, A. Ventilator-Associated Pneumonia in Neonatal and Pediatric Intensive Care Unit Patients. Clin. Microbiol. Rev. 20, 409-425 (2007).

15. El-Nawawy, A., Ramadan, M. A. F., Antonios, M. A. M., Arafa, S. A. F. \& Hamza, E. Bacteriologic profile and susceptibility pattern of mechanically ventilated paediatric patients with pneumonia. J. Glob. Antimicrob. Resist. 18, 88-94 (2019).

16. Shah, S. N., Bachur, R. G., Simel, D. L. \& Neuman, M. I. Does This Child Have Pneumonia? JAMA 318, 462 (2017).

17. Flood, R. G., Badik, J. \& Aronoff, S. C. The Utility of Serum C-Reactive Protein in Differentiating Bacterial from Nonbacterial Pneumonia in Children. Pediatr. Infect. Dis. J. PAP, 95-99 (2008).

18. Stockmann, C. et al. Procalcitonin Accurately Identifies Hospitalized Children With Low Risk of Bacterial Community-Acquired Pneumonia. J. Pediatric Infect. Dis. Soc. 7, 46-53 (2018).

19. Alejandre, C. et al. Procalcitonin-guided protocol decreased the antibiotic use in paediatric patients with severe bronchiolitis. Acta Paediatr. 109, 1190-1195 (2020).

20. Septimus, E., Green, L. \& Klompas, M. Ventilator-Associated Events. Crit. Care Med. 43, e59-e61 (2015).

21. Bradley, J. S. et al. The management of community-acquired pneumonia in infants and children older than 3 months of age: Clinical practice guidelines by the pediatric infectious diseases society and the infectious diseases society of America. Clin. Infect. Dis. 53, 25-76 (2011). 
22. Pollack, M. M., Patel, K. M. \& Ruttimann, U. E. PRISM III: an updated pediatric risk of mortality score. Crit. Care Med. 24, 743-752 (1996).

23. Magiorakos, a et al. Bacteria: an International Expert Proposal for Interim Standard Definitions for Acquired Resistance. Clin. Microbiol. Infect. 18, 268-281 (2012).

24. Davidson J, Tong S, Hancock H, Hauck A, Da Cruz E, K. J. Prospective validation of the vasoactiveinotropic score and correlation to short-term outcomes in neonates and infants after cardiothoracic surgery. Intensive Care Med 38, 1184-1190 (2012).

25. Karataş, M., Saylan, S., Kostakoğlu, U. \& Yılmaz, G. An Assessment of Ventilator-associated Pneumonias and Risk Factors Identified in the Intensive Care Unit. Pakistan J. Med. Sci. 32, 817-822 (2016).

26. Peña-López, Y. et al. Implementing a care bundle approach reduces ventilator-associated pneumonia and delays ventilator-associated tracheobronchitis in children: differences according to endotracheal or tracheostomy devices. Int. J. Infect. Dis. 52, 43-48 (2016).

27. Girona-Alarcón, M. et al. Device-associated multidrug-resistant bacteria surveillance in critically ill children: 10 years of experience. Acta Paediatr. (2020). doi:10.1111/apa.15342

28. Segers, P., Speekenbrink, R. G. H., Ubbink, D. T., van Ogtrop, M. L. \& de Mol, B. A. Prevention of Nosocomial Infection in Cardiac Surgery by Decontamination of the Nasopharynx and Oropharynx With Chlorhexidine Gluconate. JAMA 296, 2460 (2006).

29. Bouza, E. et al. Pre-emptive broad-spectrum treatment for ventilator-associated pneumonia in highrisk patients. Intensive Care Med. 39, 1547-1555 (2013).

30. Klompas, M. et al. Strategies to Prevent Ventilator-Associated Pneumonia in Acute Care Hospitals: 2014 Update. Infect. Control Hosp. Epidemiol. 35, 915-936 (2014).

31. Kollef, M. H. et al. Epidemiology and Outcomes of Health-care-Associated Pneumonia. Chest 128, 3854-3862 (2005).

32. Leung, A. K. C., Wong, A. H. C. \& Hon, K. L. Community-Acquired Pneumonia in Children. Recent Pat. Inflamm. Allergy Drug Discov. 12, 136-144 (2018).

33. Ouldali, N. et al. Long-term Association of 13-Valent Pneumococcal Conjugate Vaccine Implementation with Rates of Community-Acquired Pneumonia in Children. JAMA Pediatr. 173, 362370 (2019).

34. Katz SE, W. D. Pediatric Community-Acquired Pneumonia in the United States. Changing Epidemiology, Diagnostic and Therapeutic Challenges, and Areas for Future Research. Infect Dis Clin NAm 32, (47-63) (2018).

35. le Roux, D. M. \& Zar, H. J. Community-acquired pneumonia in children - a changing spectrum of disease. Pediatr. Radiol. 47, 1392-1398 (2017).

36. Bassetti, M., Peghin, M., Carnelutti, A. \& Righi, E. How Should We Treat HAP/VAP Caused by Carbapenemase-Producing Enterobacteriaceae? Semin. Respir. Crit. Care Med. 38, 301-310 (2017). 
37. Kollef, M. H. What Is Ventilator-Associated Pneumonia and Why Is It Important? Respir. Care 50 (6), 714-724 (2005).

38. Timsit, J.-F., Pilmis, B. \& Zahar, J.-R. How Should We Treat Hospital-Acquired and VentilatorAssociated Pneumonia Caused by Extended-Spectrum $\beta$-Lactamase-Producing Enterobacteriaceae? Semin. Respir. Crit. Care Med. 38, 287-300 (2017).

\section{Tables}

Table 1. Patient characteristics, and risk factors. 


\begin{tabular}{|c|c|c|c|c|}
\hline & General $(n=238)$ & CAP $(n=163)$ & $\operatorname{VAP}(n=75)$ & $\mathbf{p}$ \\
\hline Males, n (\%) & $125(52.5)$ & $81(49.7)$ & $44(58.7)$ & 0.211 \\
\hline Age (months) & $6.6(1.6-46.4)$ & $6.2(1.4-51.2)$ & $6.9(1.8-28)$ & 0.870 \\
\hline \multicolumn{5}{|l|}{ Risk factors, n (\%) } \\
\hline Comorbidity & $100(42)$ & $52(31.9)$ & $48(64)$ & $<0.001$ \\
\hline Tracheostomy & $8(3.4)$ & $2(1.2)$ & $6(8)$ & 0.013 \\
\hline Reason for admission, $\mathrm{n}(\%)$ & & & & 0.002 \\
\hline Respiratory & $150(63)$ & $115(70.6)$ & $35(46.7)$ & $<0.001$ \\
\hline Cardiovascular & $23(9.7)$ & $8(4.9)$ & $15(20)$ & $<0.001$ \\
\hline Neurologic & $23(9.7)$ & $14(8.6)$ & $9(12)$ & 0.408 \\
\hline Surgical & $8(3.4)$ & $6(3.7)$ & $2(2.7)$ & 1.000 \\
\hline Sepsis & $10(4.2)$ & $7(4.3)$ & $3(4)$ & 1.000 \\
\hline Oncological & $1(0.4)$ & $1(0.6)$ & $0(0)$ & 1.000 \\
\hline Other & $23(9.7)$ & $12(7.4)$ & $11(14.7)$ & 0.076 \\
\hline PRISM III & $4(2-7)$ & $4(2-7)$ & $5(2-9)$ & 0.079 \\
\hline \multicolumn{5}{|l|}{ Risk factors until infection } \\
\hline Days from admission to intubation & $0(0-0)$ & $0(0-0)$ & $0(0-2)$ & 0.002 \\
\hline Days from admission to infection & $3(1-8)$ & $1(0-3)$ & $11(7-14)$ & $<0.001$ \\
\hline Days from intubation to infection & $2(0-8)$ & $1(0-3)$ & $8(6.5-12)$ & $<0.001$ \\
\hline \multicolumn{5}{|l|}{ Biomarkers } \\
\hline C-reactive protein (mg/L) & $89(45-159)$ & $86.6(40-152)$ & $103(54-167)$ & 0.243 \\
\hline Procalcitonin (ng/mL) & $1.35(0.35-6.1)$ & $1.3(0.4-5.7)$ & $1.7(0.3-7.9)$ & 0.850 \\
\hline Lactate $(\mathrm{mmol} / \mathrm{L})$ & $2.3(1.8-3)$ & $2.2(1.8-2.9)$ & $2.4(2-3)$ & 0.191 \\
\hline \multicolumn{5}{|l|}{ Treatment } \\
\hline Inotropic treatment, n (\%) & $87(36.6)$ & $50(30.7)$ & $37(49.3)$ & 0.006 \\
\hline Inotropic length (days) & $5(2-9)$ & $3(2-5)$ & $8(4-13)$ & $<0.001$ \\
\hline Maximum VIS & $10(5.5-29.5)$ & $10(5-28)$ & $15(10-30)$ & 0.073 \\
\hline Antibiotic length (days) & $7(7-10)$ & $7(7-8)$ & $10(7-13)$ & $<0.001$ \\
\hline Antibiotic switched, n (\%) & $121(50.8)$ & $74(45.7)$ & $47(63.5)$ & 0.011 \\
\hline
\end{tabular}


Values are expressed as frequency (percentage) for qualitative variables and as median (interquartile range) for quantitative variables. PRISM III: Paediatric Risk Mortality III score. CAP: Community-acquired pneumonia. VAP: Ventilator-associated pneumonia. VIS: Vasoactive Inotropic Score.

Table 2. Microbiological data of the respiratory samples. 


\section{$\operatorname{CAP}(n=163) \quad \operatorname{VAP}(n=75) \quad p$}

\section{Respiratory cultures}

Type of microorganism

$\begin{array}{lllll}- & \text { Gram positive } & 60(36.8) & 6(8) & <0.001 \\ -\quad \text { Gram negative } & 94(57.7) & 33(44) & 0.05 \\ -\quad \text { Enterobacteriaceae } & 9(5.5) & 36(48) & <0.001 \\ \text { Resistance pattern } & & & \\ -\quad \text { DS } & 126(77.3) & 55(73.3) & 0.505 \\ -\quad \text { Non-DS } & 37(22.6) & 20(26.6) & \\ -\quad \text { DR } & 32(86.5) & 13(65) & 0.058 \\ -\quad & 4(10.8) & 6(30) & 0.141 \\ -\quad \text { MDR } & 1(2.7) & 1(5) & 1.000 \\ -\quad \text { XDR } & 4(10.8) & 2(10) & 1.000 \\ -\quad \text { MRSA } & 3(8.1) & 6(30) & 0.054\end{array}$

\section{Specific microorganisms}

\begin{tabular}{|lllll|} 
Gram positive & Enterococcus spp. & $1(0.6)$ & $1(1.3)$ & 0.532 \\
& S. aureus & $27(16.6)$ & $4(5.3)$ & 0.021 \\
& S. pneumoniae & $28(17.2)$ & $0(0)$ & $<0.001$ \\
& S. pyogenes & $3(1.8)$ & $0(0)$ & 0.554 \\
& S. viridans & $1(0.6)$ & $1(1.3)$ & 0.532 \\
Gram negative & A. xylosoxidans & $0(0)$ & $1(1.3)$ & 0.315 \\
& B. cepacia & $0(0)$ & $2(2.7)$ & 0.098 \\
& Haemophilus spp. & $66(40.5)$ & $5(6.7)$ & $<0.001$ \\
& M. catarrhalis & $22(13.5)$ & $2(2.7)$ & 0.010 \\
& N. meningitidis & $1(0.6)$ & $0(0)$ & 1.000 \\
& P. aeruginosa & $5(3.1)$ & $17(22.7)$ & $<0.001$ \\
& P. mirabilis & $0(0)$ & $1(1.3)$ & 0.315 \\
& S. maltophilia & $0(0)$ & $5(6.7)$ & $\mathbf{0 . 0 0 3}$ \\
\hline Enterobacter spp. & $2(1.2)$ & $12(16)$ & $<0.001$ \\
& E. coli & $3(1.8)$ & $6(8)$ & $\mathbf{0 . 0 3 0}$
\end{tabular}




\begin{tabular}{|llll|} 
Klebsiella spp. & $4(2.5)$ & $13(17.3)$ & $<0.001$ \\
M. morgannii & $0(0)$ & $1(1.3)$ & 0.315 \\
Serratia spp. & $0(0)$ & $4(5.3)$ & $\mathbf{0 . 0 0 9}$ \\
\hline
\end{tabular}

Values are expressed as frequency (percentage) for qualitative variables. CAP: Community-acquired pneumonia. VAP: Ventilator-associated pneumonia. DS: Drug-sensitive. Non-DS: Non-drug-sensitive. DR: Drug-resistant. MDR: Multidrug-resistant. XDR: Extensively drug-resistant. MRSA: Methicillin-resistant staphylococcus aureus. ESBL: Extended spectrum beta-lactamases.

Table 3. Comparison between Community-acquired pneumonia and ventilator-associated pneumonia regarding the outcomes.

\begin{tabular}{|lllll|}
\hline Outcomes & General $(\mathbf{n = 2 3 8})$ & CAP $(\mathbf{n = 1 6 3 )}$ & VAP $(\mathbf{n = 7 5 )}$ & $\mathbf{p}$ \\
\hline Days on MV & $9(6-14)$ & $7(4.7-12)$ & $14(11-21)$ & $<0.001$ \\
Days on NIV & $2(0-5)$ & $2(0-4)$ & $3(1-8)$ & $<0.001$ \\
Length of stay in ICU (days) & $15(9-22)$ & $12(7-16)$ & $24(18-38)$ & $<0.001$ \\
Length of stay in hospital (days) & $22(15-34)$ & $19(14-26)$ & $35.5(26-57.3)$ & $<0.001$ \\
Sepsis, n (\%) & $42(17.6)$ & $24(14.7)$ & $18(24)$ & 0.081 \\
Positive blood culture, n (\%) & $31(13)$ & $18(11)$ & $13(17.3)$ & 0.180 \\
ECMO needed, n (\%) & $11(4.6)$ & $3(1.8)$ & $8(10.7)$ & $\mathbf{0 . 0 0 5}$ \\
Mortality, $\mathrm{n}(\%)$ & $13(5.5)$ & $4(2.5)$ & $9(12)$ & $\mathbf{0 . 0 0 5}$ \\
\hline
\end{tabular}

Values are expressed as frequency (percentage) for qualitative variables and as median (interquartile range) for quantitative variables. CAP: Community-acquired pneumonia. VAP: Ventilator-associated pneumonia. MV: Mechanical ventilation. NIV: Non-invasive mechanical ventilation. ICU: Intensive care unit. ECMO: Extracorporeal membrane oxygenation. 